\section{GH/IGF e Neoplasia: O Que Há de Novo Nesta Associação}

\section{RESUMO}

Estudos in vitro e em animais sugerem que os membros do sistema insulin-like growth factors (IGFs), incluindo IGF-I, IGF-II, receptores de IGFI e IGF-II (IGF-IR e IGF-IIR), e as IGF-binding proteins (IGFBPs) podem ter um importante envolvimento no desenvolvimento e na progressão de neoplasias. Mais especificamente, as IGFs promovem a progressão do ciclo celular e inibem a apoptose tanto por ação direta com outros fatores de crescimento como por ação indireta interagindo com outros sistemas moleculares intracelulares envolvidos na promoção e/ou progressão do câncer. Além disso, inúmeros estudos epidemiológicos têm sugerido que concentrações elevadas das IGFs, independente das alterações nas IGFBPs, podem estar associadas a um aumento no risco de desenvolver determinadas neoplasias. Esta revisão tem como objetivo apresentar o envolvimento do sistema IGF na regulação tumoral, os principais estudos epidemiológicos realizados e o risco de desenvolvimento de neoplasia em pacientes (com ou sem história pessoal de neoplasia prévia) que receberam hormônio de crescimento (rhGH). É importante salientar que o uso clínico de rhGH, nas indicações aprovadas internacionalmente, é seguro e não existem evidências, até o momento, da associação com o desenvolvimento de neoplasias. (Arq Bras Endocrinol Metab 2005;49/5:833-842)

Descritores: Hormônio de crescimento; IGF; Receptor de IGF; IGFBP; Neoplasia; Estudo epidemiológico; Meta-análise

\begin{abstract}
GH/IGF-1 and Cancer: What's New in this Association.

Evidence from in vitro and animal studies suggests that members of the insulin-like growth factors (IGFs) system, including IGF-I, IGF-II, the IGF-I receptor (IGF-IR), and the IGF-binding proteins (IGFBPs) play a central role in the development and progression of cancer. More specifically, IGFs may promote cell cycle progression and inhibition of apoptosis either by directly associating with other growth factors or indirectly by interacting with other molecular systems, which have an established role in carcinogenesis and cancer promotion. In addition, a growing number of epidemiologic studies suggest that increased serum levels of IGFs and/or altered levels of their binding proteins are associated with increased risk for developing several malignancies. This review aims to summarize and to show the role of IGF system in tumor regulation, a revision of epidemiologic studies and the risk of neoplasia in patients (with or without personal history of previous neoplasia) who received growth hormone (rhGH). It is important to emphasize that the clinical use of $\mathrm{rhGH}$, in the indications internationally approved, is secure, and there are not evidences, at this moment, of the association with neoplasias development. (Arq Bras Endocrinol Metab 2005;49/5:833-842)
\end{abstract}

Keywords: Growth hormone; IGF; IGF-receptor; IGFBP; Neoplasia; Epidemiologic study; Meta-analysis revisão Departamento de Endocrinologia
da Sociedade de Pediatria de
São Paulo, SP. 
$\mathrm{O}$ EIXO GH (GROWTH HORMONE) - IGF (insulin-like growth factor) tem um papel bem estabelecido na regulação do crescimento somático, e muitas são as evidências de que esses hormônios também contribuem para o crescimento de tecidos neoplásicos. Diversos estudos experimentais e populacionais procuram estabelecer uma relação entre as concentrações de IGF-I, IGFBP3 (insulin-like growth factor binding pro tein) e o risco de câncer (1-4).

Durante o período em que o hormônio de crescimento (proveniente de hipófises humanas) tinha uma indicação restrita aos pacientes com comprovada deficiência, já existia a preocupação com a avaliação dos efeitos adversos desse tratamento. Com a introdução do hormônio recombinante ( $\mathrm{rhGH}$ ), surgiram novas indicações terapêuticas, que hoje englobam pacientes com síndrome de Turner, insuficiência renal crônica, retardo de crescimento intra-uterino, baixa estatura idiopática, síndrome de Prader-Willi, além da deficiência de GH durante a fase adulta. Modificou-se também o tempo de uso, a dose e o esquema terapêutico (5). Devido ao grande número de pacientes tratados, a preocupação com os efeitos adversos tornou-se ainda maior, levando à realização de diversos estudos multicêntricos, pesquisas e consensos, estimulando um debate maior a respeito dos possíveis riscos, procurando criar condições para tornar o seu uso mais seguro $(6,7)$.

A primeira evidência de uma possível associação entre GH e câncer surgiu em 1950, com a demonstração de que doses suprafisiológicas do $\mathrm{GH}$, quando administradas a ratos, causavam alterações neoplásicas em vários órgãos, enquanto a hipofisectomia revertia um quadro de leucemia ou protegia o animal de desenvolver a doença $(8)$.

Posteriormente, outros estudos demonstraram a atividade mitogênica do GH e os seus efeitos tróficos no tecido linfóide. Em 1977, Rogers e cols. levantaram a hipótese do envolvimento do $\mathrm{GH}$ e da somatomedina (IGF-I) na etiopatogenia da leucemia linfóide aguda, após detectar em crianças leucêmicas alterações desses hormônios de acordo com a fase de atividade ou remissão da doença (9).

Vários estudos foram feitos, demonstrando que o GH, devido ao seu efeito metabólico e mitogênico, e a IGFl, pelo seu efeito anti-apoptótico, agem na proliferação das células normais e malignas, via receptor de IGF-I, e são responsáveis pela indução de tumores em animais expostos a doses suprafisiológicas do $\mathrm{GH}$, induzindo in vitro à transformação de células hematopoiéticas normais em células leucêmicas. Estudos experimentais recentes indicam, por um lado, que o GH pode produzir tumores ativos, o que seria uma contra-indicação para o seu uso, porém, também é considerado benéfico na redução da caquexia dos pacientes com câncer, melhorando a sobrevivência e justificando sua indicação (1-4,6,7).

Quando a relação entre o uso do rhGH e o aparecimento de neoplasias é analisada, duas questões se colocam. A primeira é se o rhGH induz ao aparecimento de tumores em pacientes sem doença neoplásica, e a segunda é se pode influenciar na recidiva da doença tumoral, em pacientes considerados curados ou em remissão. O objetivo do presente artigo é rever o que há de novo nessa associação, tanto do ponto de vista fisiológico como epidemiológico e clínico.

\section{Fisiologia do sistema IGF}

O sistema IGF inclui os ligantes IGF-I e IGF-II, os receptores (tipo 1 e 2 de IGF, IGF-IR e IGF-IIR, respectivamente), as proteínas ligadoras de IGF (IGFBPI a 6) e as proteínas intracelulares sinalizadoras associadas ao IGF-IR, que incluem os membros da família do IRS (insulin-receptor substrate), AKT, TOR (target of rapamicina) e a S6K (S6 kinase) (10-13).

$\mathrm{O}$ fígado é o responsável pela síntese da maior concentração circulante de IGFs e IGFBPs, mas essas substâncias também podem ser localmente secretadas em outros órgãos por ação autócrina ou parácrina (13). O GH é o principal estimulador da produção de IGF-I, mas não da IGF-II. A IGF-I apresenta concentrações sistematicamente aumentadas, em paralelo com o crescimento pós-natal e puberal, declinando após a interrupção do crescimento e voltando a aumentar na velhice. A IGF-II está expressa em altas concentrações nos embriões, mas após o nascimento continua a ser expressa e secretada pelo fígado, estando presente durante toda a vida (14).

As IGFs são transportadas para as células-alvo em complexo com as IGFBPs, diferentemente da insulina, o que prolonga sua meia-vida e modula sua interação com a superfície de membrana dos receptores. A IGFBP3, entre as IGFBPs, é a forma circulante mais abundante, responsável pela maior parte da capacidade de ligação às IGFs, em especial à IGF-I; e está normalmente associada a um complexo ternário composto por ela, a IGF-I e a ALS (acid-labile sub unit). As IGFBPl e IGFBP6 possuem uma capacidade de ligação 10 vezes maior à IGF-II em relação à IGFI, enquanto as demais IGFBPs $(10,11)$ têm igual afinidade às IGFs. Nenhuma das IGFBPs se liga especificamente à insulina (10-15).

As principais diferenças entre as IGFs e a insulina estão na estrutura, nos receptores preferenciais, nas interações com as proteínas ligadoras, e, conseqüente- 
mente, na função. A insulina age como um ligante solto para ativar o seu receptor (IR), enquanto as IGFI e II, uma vez ligadas às IGFBPs, ativam predominantemente o IGF-IR, que tem estrutura semelhante ao IR. IGFs e insulina, embora esta última com menor afinidade, têm a capacidade de se ligar a um receptor híbrido de IGF-IR e IR. A IGF-II se liga também a uma isoforma A relacionada ao IR (insulin related IR$A)$ e ao receptor IGF-IIR (também chamado de receptor de IGF-II/manose 6-fosfato). A principal função do IGF-IIR é ser um regulador negativo da biodisponibilidade da IGF-II (12,13,15-19).

Tanto a parte justa-membranosa intracelular como o domínio C-terminal dos receptores IGF-IR e IR possuem um menor número de seqüências idênticas que o domínio tirosina-quinase, o que lhes confere especificidade. Após a ligação da IGF-I ou da IGF-II ao IGF-IR ocorre uma atividade catalítica do domínio tirosina-quinase que desencadeia a fosforilação de substratos intracelulares (figura 1) $(17,20,21)$.

As proteínas IRS (insulin receptor substrate) 1 a $4 \mathrm{e}$ as Shc (Src homology collagen) constituem a maioria dos substratos para a fosforilação intracelular. A IRS fosforilada recruta a PI3K (phosphatidylinositol 3kinase), resultando em ativação da PDK (phosphoinosi tide-dependent protein kinase), $\mathrm{PKB}$ (protein kinase B) e p70S6 quinase, que em cascata irão controlar a transcrição via FOXO (forkhead transcription factors), o metabolismo via GSK-3 $\beta$ (glycogen synthetase kinase $3 \beta$ ), a apoptose via BAD (bcl-associated death promot er), o crescimento celular via TOR e a translação via
eIF4E (eukaryotbic initiation factor 4E)-S6K. A ativação da proliferação celular é mediada pela ligação da proteína SH2 ao adaptador Grb2 (growth factor recep tor bound protein 2) do receptor, o que desencadeia a ativação de SOS, Ras, Raf (GTPase-mediated proteins family) e MAPK (mitogen-activated protein kinase). Reguladores negativos deste sistema incluem a Grb2, o sistema JAK (janus tyrosine kinase) e a PTEN (phos phatase mutated on chromosome 10) (figura 1) (22-27).

A ligação da IGF-II com o seu respectivo receptor (IGF-IIR) não desencadeia sinal intracelular por não ter o domínio tirosina-quinase, e parece ter ação negativa na proliferação pela redução das quantidades de IGF-II viáveis à ligação ao $\operatorname{IGF-IR~(21).~}$

\section{IGF e desenvolvimento de neoplasia}

Estudos experimentais mostram que o crescimento de neoplasias que expressam IGF-IR é estimulado pela IGFI sintetizada em tecidos que podem estar muito distantes destas neoplasias. No entanto, outras neoplasias são também influenciadas pela produção autócrina ou parácrina de IGFs. A progressão da neoplasia pode ser caracterizada por uma fase inicial na qual os cânceres ainda dependem dos ligantes IGFs antes de se tornarem capazes de produzi-los de maneira autócrina. Esta capacidade de se auto-estimular pode estar associada ao comportamento mais agressivo da neoplasia (1-3,28).

A expressão de IGF-II na progressão de neoplasia pode ser vista no câncer de cólon, pois o gene da IGF-II é o que mais se expressa nestas neoplasias em comparação à mucosa do cólon normal, e a perda de

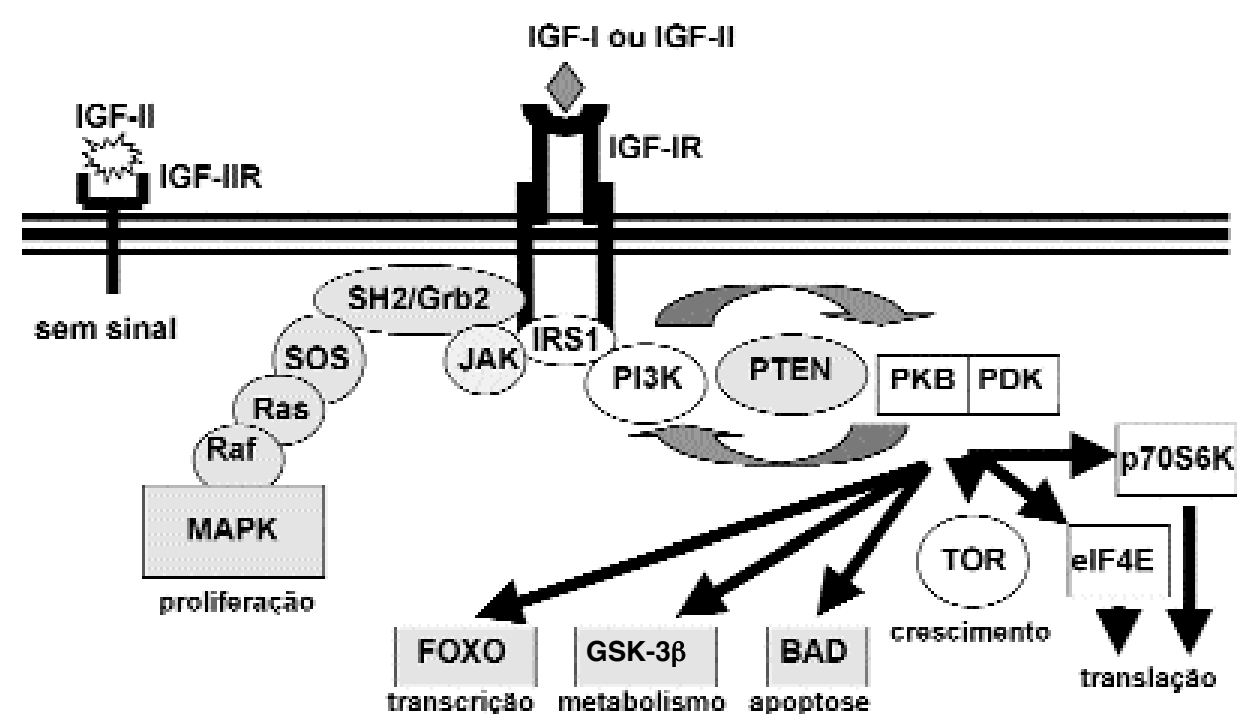

Figura 1. Representação simplificada dos componentes intracelulares do sistema IGF e suas ações. 
imprinting da IGF-II também representa um fator de risco para esta neoplasia (29-31).

Há grandes evidências de uma associação entre a progressão da neoplasia e o aumento da expressão de IGF-IR, particularmente no câncer de próstata. O IGF-IIR também tem participação no desenvolvimento da neoplasia, pois quando é perdido ocorre um aumento na ativação do IGF-IR pela IGF-II, o que sugere que o IGF-II possa ter propriedades de um gene supressor tumoral $(31,32)$.

O modelo mais simples para explicar a ação das IGFBPs na neoplasia postula que elas modulam a biodisponibilidade das IGFs na circulação e no espaço extracelular por competição com receptores pelos ligantes. Quando as concentrações de IGFBPs estão diminuídas, devido aos efeitos das proteases presentes nos tumores que digerem as IGFBPs, a atividade mitogênica pode aumentar. Portanto, a liberação de proteases pelos cânceres aumentaria a sinalização ao IGF-IR. Entretanto, de forma menos clara, algumas IGFBPs têm efeito inibitório no crescimento independente de IGF, ou efeito pró-apoptótico, ou as células tumorais podem se tornar resistentes a essas ações. Além disso, sob determinadas situações, as IGFBPs parecem potencializar a atividade mitogênica das IGFs (12,33-37).

\section{Concentraçōes séricas de IGF e risco de câncer}

As concentrações circulantes de IGF-I, IGF-II e IGFBPs variam entre indivíduos normais, influenciadas por fatores genéticos e estilo de vida, em especial a alimentação. Estudos populacionais têm revelado risco elevado de câncer nos indivíduos com concentrações de IGF-I no limite superior do normal (tabela 1). Estes dados são confirmados com estudos de carcinogênese in vivo, mostrando aumento da taxa de divisão celular e efeito anti-apoptótico da IGF-I. A diminuição da IGF-I diminui as condições permissivas para a proliferação neoplásica.
Na tabela 1 são apresentados os dados do estudo caso-controle realizado por Chan e cols. em 1998, nos Estados Unidos, com 15.000 homens entre 40 e 82 anos de idade, com 152 casos de câncer de próstata, sendo a IGF-I dosada em média 7 anos antes do diagnóstico. Neste estudo, observa-se o risco relativo alto para o câncer quando se tem apenas IGF-I aumentada $(\mathrm{RR}=$ $2,4)$, ou associada a IGFBP3 $(\mathrm{RR}=4,3)$ ou ainda ambas aumentadas em pacientes acima de 60 anos $(\mathrm{RR}=7,9)$ (38). Hankinson e cols., em 1998, também realizaram um estudo caso-controle com 121.700 mulheres entre 30 e 55 anos de idade, com 397 casos confirmados de câncer de mama e com IGF-I dosada em média 28 meses antes do diagnóstico. Neste estudo, observa-se o risco relativo alto para o câncer quando se tem apenas IGF-I aumentada $(\mathrm{RR}=2,3)$, ou na pré-menopausa em idade abaixo dos 50 anos $(\mathrm{RR}=4,6)$, ou ainda associada a IGFBP3 ( $\mathrm{RR}=7,3)(39)$.

As neoplasias de próstata e mama são geralmente dependentes de hormônios sexuais. Para o câncer de próstata, a associação ocorre com os andrógenos. Gann e cols., em 2 estudos, mostraram o risco relativo desta neoplasia na presença do antígeno prostático específico $(\mathrm{RR}=5,5)(40)$ e quando valores elevados de testosterona e da proteína ligadora de hormônios sexuais (SHBG) estavam elevados $(\mathrm{RR}=2,6)(4 \mathrm{l})$. O mesmo foi feito para o câncer de mama com os estudos de Dorgan e cols. em 1997 (42), mostrando o risco relativo na pós-menopausa na presença de valores elevados de estradiol e SHBG $(\mathrm{RR}=5,2)$ e de testosterona $(\mathrm{RR}=$ 6,2). Os estudos de Harris e cols. em 1996 mostraram os riscos relativos de câncer de mama na presença de uso de contraceptivo oral como terapia de reposição hormonal $(\mathrm{RR}=1,0$ a 2,0$)$ ou com história de menarca precoce, menopausa tardia, nuliparidade ou idade na primeira gestação $(\mathrm{RR}=1,5$ a 3,0) (43).

Nos pacientes adultos com deficiência de GH, ressalta-se um aspecto importante, qual seja, o fato de que a IGF-I está normal em 50\% dos casos, o que significa

Tabela 1. IGF-I como fator de risco para câncer de próstata e mama.

\begin{tabular}{lcc}
\hline & Risco Relativo & Comparação \\
\hline Câncer de Próstata (33) & & \\
IGF-I & 2,4 & \\
IGF-I e IGFBP 3 & 4,3 & Quartil superior x inferior \\
IGF-I, IGFBP3 e idade > 60 anos $\quad 7,9$ & \\
Câncer de Mama (34) & 2,3 & Tercil superior x inferior \\
IGF-I e idade < 50 anos e pré-menopausa & & \\
IGF-I, IGFBP3, idade < 50 anos e pré-menopausa & & \\
\hline
\end{tabular}


que após a introdução da medicação esses pacientes terão seus valores no limite superior da normalidade ou acima desse. Para alguns, a dose de rhGH suficiente para normalizar a IGF-I não corrige as alterações do metabolismo intermediário. $\mathrm{O}$ quanto esse aumento implica em um risco adicional para o desenvolvimento de câncer de mama, próstata, colo-retal ou outros, são preocupações à luz dos estudos epidemiológicos (44). Por outro lado, essa reposição pode ser muito necessária para melhorar as condições cardíacas e nutricionais.

Até o momento não existem evidências de que o uso de rhGH em situações clínicas aprovadas internacionalmente e em doses adequadas esteja associado ao desenvolvimento de neoplasias.

\section{Estudos epidemiológicos}

Os estudos mostrando uma correlação entre as concentrações elevadas de IGF-I e baixas de IGFBP-3 e o risco aumentado de neoplasias, realizados por Chan e cols., em 1998, em homens com câncer de próstata, e por Hankinson e cols., em 1999, em mulheres com câncer de mama antes da menopausa, desencadearam inúmeras outras pesquisas nesta área (tabela 1 ).
Em 2004, Renehan e cols. (45) realizaram uma ampla revisão de todos os trabalhos publicados na literatura entre janeiro de 1996 e dezembro de 2002, envolvendo IGF-I e IGFBP3 e o risco de câncer de próstata, de cólon, de mama e de pulmão. Entre 139 estudos identificados, apenas 21 foram escolhidos por preencherem os critérios adequados para avaliação por meta-análise (tabela 2 ). Por meio de uma análise doseresposta, este estudo mostrou que o aumento nas concentrações de IGF-I se associa apenas a um risco aumentado para as neoplasias de próstata e de mama na pré-menopausa, enquanto que a IGFBP3 associa-se à neoplasia de mama pré-menopausa (tabela 3 ).

Do ponto de vista epidemiológico, é importante salientar que os dados de IGF-I são mais relevantes que os de IGFBP3, e que alguns estudos não detectam associação de uma determinada neoplasia com IGF-I e/ou IGFBP3 (45), pois este risco se torna insignificante quando comparado a outros fatores que podem levar à carcinogênese, como a associação entre neoplasia de pulmão e fumo (63). A associação entre IGF-I e câncer de próstata entre fumantes e não-fumantes não é observada no grupo dos fumantes (65).

Tabela 2. Comparação dos valores mais altos e mais baixos de IGF-I e IGFBP3 por câncer.

\begin{tabular}{|c|c|c|c|c|}
\hline \multirow{2}{*}{$\begin{array}{l}\text { Estudo } \\
\text { Câncer de Próstata }\end{array}$} & Casos/Controles & Categoria & \multicolumn{2}{|c|}{ Risco Relativo } \\
\hline & & & & \\
\hline Chan e cols. $(1998)^{*}(38)$ & $152 / 152$ & Quartis & 4,32 & 0,41 \\
\hline Harman e cols. $(2000)^{*}(46)$ & $72 / 203$ & Tercis & 3,11 & 0,76 \\
\hline Stattin e cols. $(2000) *(47)$ & $149 / 298$ & Quartis & 1,32 & 1,19 \\
\hline Wolk e cols. (1998)\# (48) & $224 / 224$ & Quartis & 1,43 & 1,21 \\
\hline Finne e cols. (2000)\# (49) & $179 / 486$ & Quartis & 0,57 & 1,24 \\
\hline Chokkalimgam e cols. (2001)\# (50) & $128 / 306$ & Quartis & 3,92 & 0,47 \\
\hline \multicolumn{5}{|l|}{ Câncer de Cólon } \\
\hline Ma e cols. (1999)* (51) & $193 / 318$ & Quintis & 2,51 & 0,28 \\
\hline Giovannucci e cols. $(2000)^{\star}(52)$ & $79 / 158$ & Tercis & 2,18 & 0,28 \\
\hline Kaaks e cols. $(2000)^{*}(53)$ & $102 / 200$ & Quintis & 1,23 & 1,23 \\
\hline Probst-Hensch e cols. $(2001) *(54)$ & $135 / 661$ & Quintis & 1,18 & 1,78 \\
\hline Palmkvist e cols. $(2002)^{\star}(55)$ & $168 / 336$ & Quartis & 1,27 & 1,32 \\
\hline \multicolumn{5}{|l|}{ Câncer de Mama - pré-menopausa } \\
\hline Hankinson e cols. (1999)* (39) & $76 / 105$ & Tercis & 2,88 & - \\
\hline Toniolo e cols. $(2000) *(56)$ & $172 / 486$ & Quartis & 1,60 & 1,18 \\
\hline Muti e cols. $(2002) *(57)$ & $69 / 267$ & Quartis & 3,12 & 2,31 \\
\hline Krajeik e cols. $(2002)^{\star}(58)$ & $66 / 66$ & Quartis & 2,01 & 5,28 \\
\hline Del Giudice e cols. (1998)\# (59) & 99/99 & Quintis & 1,47 & 2,05 \\
\hline Yu e cols. (2002)\# (60) & $178 / 170$ & Tercis & 1,92 & 2,69 \\
\hline \multicolumn{5}{|l|}{ Câncer de Mama - pós-menopausa } \\
\hline Hankinson e cols. $(1998)^{\star}(39)$ & $305 / 483$ & Tercis & 0,89 & - \\
\hline Toniolo e cols. $(2000) *(56)$ & $115 / 220$ & Quartis & 0,95 & 1,08 \\
\hline Muti e cols. $(2002)^{*}(57)$ & $64 / 238$ & Quartis & 0,58 & 0,73 \\
\hline Krajeik e cols. $(2002) *(58)$ & $60 / 60$ & Quartis & 1,22 & 0,32 \\
\hline Yu e cols. (2002)\# (60) & $128 / 130$ & Tercis & 1,56 & 2,11 \\
\hline \multicolumn{5}{|l|}{ Câncer de Pulmão } \\
\hline Lukanova e cols. (2001)* (61) & $93 / 186$ & Quartis & 0,54 & 0,90 \\
\hline London e cols. $(2002) *(62)$ & $230 / 740$ & Quartis & 0,86 & 0,50 \\
\hline Spitz e cols. $(2002) *(63)$ & $159 / 397$ & Quartis & 0,64 & 2,35 \\
\hline Yu e cols. (1999)\# (64) & $204 / 218$ & Quartis & 2,75 & 0,48 \\
\hline
\end{tabular}

* = estudo tipo coorte; \# = estudo tipo caso-controle 
Tabela 3. Meta-análise por dose resposta da associação de IGF-I e IGFBP3 com o tipo de neoplasia.

\begin{tabular}{lccr}
\hline & Estudos & Risco Relativo (95\% IC) & p \\
\hline IGF-I & & & \\
Próstata & $3(47,48,50)$ & $1,49(1,14-1,95)$ & 0,003 \\
Cólon & $4(52-55)$ & $1,18(0,92-1,51)$ & 0,19 \\
Mama pré-menopausa & $4(39,56,57,60)$ & $1,65(1,26-2,08)$ & $<0,001$ \\
Mama pós-menopausa & $4(39,56,57,60)$ & $0,95(0,77-1,17)$ & 0,63 \\
Pulmão & $4(61-64)$ & $1,01(0,65-1,58)$ & 0,95 \\
IGFBP3 & $3(47,48,50)$ & $0,95(0,70-1,28)$ & 0,72 \\
Próstata & $4(52-55)$ & $1,16(0,85-1,57)$ & 0,35 \\
Cólon & $3(56,57,60)$ & $1,51(1,01-2,27)$ & 0,05 \\
Mama pré-menopausa & $3(56,57,60)$ & $1,01(0,74-1,38)$ & 0,93 \\
Mama pós-menopausa & $4(61-64)$ & $0,98(0,62-1,54)$ & 0,92 \\
Pulmão & & & \\
\hline
\end{tabular}

$\mathrm{IC}=$ intervalo de confiança

A relação entre IGF-I e IGFBP foi também avaliada em outras doenças. Juul e cols., em 2002, demonstraram, de forma inversa ao observado nas neoplasias, que indivíduos com IGF-I baixa e IGFBP-3 elevada apresentam risco aumentado $(\mathrm{RR}=4,07)$ de desenvolverem doença isquêmica cardíaca durante um período de 15 anos de acompanhamento (66). Sandhu e cols., em 2002, mostraram que concentrações baixas de IGFI e IGFBP-1 associadas representam risco para desenvolvimento de intolerância a glicose ou diabetes mellitus tipo 2, após um acompanhamento de 4 anos e meio de 615 indivíduos normoglicêmicos inicialmente (67).

\section{Risco de neoplasia em pacientes tratados com rhGH}

Sem história de neoplasia prévia: O interesse em determinar se o GH pode desencadear uma neoplasia é muito antigo. Vários estudos clínicos foram feitos para avaliar se existe um risco dessa associação. Em 1988, Watanabe descreveu um aumento na incidência de leucemia em crianças japonesas durante o tratamento com $\mathrm{GH}$ e, nessa mesma época, a Lawson-Wilkins Society for Pedi atric Endocrinology fez uma revisão de todos os casos semelhantes publicados, calculando o risco dos pacientes em uso de rhGH desenvolverem leucemia como sendo duas vezes maior do que o esperado para a população pediátrica $(6,7,68)$. Convém referirmos que a leucemia é a neoplasia mais freqüente na infância, afetando em média 1:2.000 crianças com idade inferior a 15 anos e, embora a maioria dos casos seja idiopática, alguns fatores de risco para a doença são conhecidos, como idade materna avançada, história familiar, síndromes congênitas, tratamento anterior com agentes citotóxicos e radioterapia (69).

A relação entre $\mathrm{GH}$ e leucemia foi reavaliada por estudos mais recentes, envolvendo um número significativo de pacientes, acompanhados por tempo prolongado, que concluíram que a ocorrência de leu- cemia ou da síndrome mielodisplásica em pacientes tratados com GH é comparável à da população geral, e não se confirmaram, portanto, os achados anteriores. No entanto, identificou-se nesses estudos um grupo de pacientes considerados de risco para desenvolver leucemia, entre os quais se incluem os portadores de anemia de Fanconi, síndromes de Down e Bloom, histiocitose, anemia aplástica e neurofibromatose, assim como os pacientes tratados previamente com radioterapia. O uso de rhGH nessa população não é aconselhado $(6,7,44,45,68-73)$.

Em relação ao desenvolvimento de outros tipos de neoplasias, apesar dos estudos epidemiológicos indicarem uma freqüência aumentada de tumor de cólon em acromegálicos, poucas pesquisas foram feitas até o momento para determinar esse risco nos recipientes de $\mathrm{GH}$, e os resultados obtidos não são concordantes. Tuffli e cols. (74) não demonstraram uma tendência de aumento nas neoplasias relacionada ao uso de GH. No entanto, Swerdlow e cols. (75) estudaram 1.848 pacientes adultos (idade inferior a 40 anos), tratados com GH humano no período de 1959 a 1985, demonstrando após um período de observação de 16 a 20 anos uma taxa de mortalidade por câncer, nesse grupo, maior do que a esperada. $\mathrm{O}$ câncer colo-retal foi o mais freqüente, seguido pela doença de Hodgkin. Esses resultados, embora sejam importantes, ainda representam dados preliminares, sem outros trabalhos semelhantes para comparação. $\mathrm{O}$ número de pacientes afetados, embora seja muito pequeno, é significante estatisticamente, visto que a incidência esperada de câncer de cólon na população estudada foi avaliado em 0,18 casos, sendo necessária muita prudência na interpretação desses resultados. Não existem evidências de casos de carcinoma colo-retal descritos com o esquema terapêutico atual, mas as comparações não são possíveis, devido à grande diferença entre os tratamentos, principalmente em relação às doses utilizadas. 
Bancos de dados internacionais que fazem um acompanhamento sistemático dos pacientes pediátricos tratados com rhGH e acumulam dados de mais de 300.000 pacientes, relatam o caso de uma paciente anteriormente tratada por tumor de sistema nervoso central que desenvolveu um tumor gastrointestinal, e uma paciente com síndrome de Turner com câncer de cólon (6,70-72).

É importante observar que em todas as pesquisas é difícil efetuar-se um cálculo adequado do risco de desenvolver leucemia ou tumores sólidos sem que se conheça a incidência de câncer nos pacientes deficientes de GH que não foram tratados. Existe, inclusive, a dúvida se a deficiência de GH per si poderia predispor ao desenvolvimento de leucemia $(6,45)$.

Em 2004, os membros da Lawson-Wilkins Soci ety for Pediatric Endocrinology elaboraram um editorial questionando se a ocorrência de neoplasia nos pacientes tratados com rhGH seria coincidência ou conseqüência, e concluíram que no momento atual os dados disponibilizados não indicam uma preocupação com malignidade, e sustentam seu posicionamento com base em um estudo feito com 6.284 pacientes tratados por deficiência idiopática de GH. Nesse grupo, embora alguns pacientes com radiação prévia tenham desenvolvido leucemia, não foi encontrado aumento significativo no número de pacientes que desenvolveram a doença (76).

$O$ risco de desenvolver uma neoplasia não parece estar aumentado nos pacientes com deficiência de GH tratados. Com a expansão nas indicações do rhGH, estima-se que mais de 100.000 pacientes estarão em tratamento nos próximos anos, de tal forma que estabelecer se a exposição precoce ao rhGH poderá influenciar a incidência de neoplasias a longo prazo continuará sendo uma preocupação.

Com história de neoplasia prévia: Nas últimas duas décadas, a porcentagem de crianças tratadas por câncer que atingem a cura aumentou de forma significativa. Devido a essa sobrevida importante, os profissionais que cuidam desses pacientes passaram a se defrontar com vários problemas, entre os quais salientam-se as alterações hormonais, como atraso de crescimento e da puberdade, hipotiroidismo e infertilidade, que têm como principal causa o tratamento quimio e radioterápico. A deficiência de $\mathrm{GH}$ é a alteração endócrina mais comum e a que provoca as principais dúvidas e questionamentos, tanto em relação ao diagnóstico quanto ao tratamento (69).

A questão principal quando se analisa a indicação do rhGH nos pacientes tratados por câncer durante a infância é se uma substância com importante capacidade de promover crescimento, um potente anabolizante, com ações mitogênicas e proliferativas, aumentaria o risco de recorrência da doença de base ou poderia desencadear o desenvolvimento de uma segunda neoplasia.

A recorrência do tumor de sistema nervoso central tem sido apontada como uma causa freqüente de óbito de pacientes tratados com rhGH. Vários estudos têm avaliado se o risco de recorrência dos tumores é modificado pelo uso de rhGH. É pertinente lembrar que para os diferentes tumores existe uma taxa de sobrevida e uma incidência esperada de recidivas, mas nem sempre essas comparações são realizadas, sendo difícil avaliar se a porcentagem de recorrência é superior ao esperado na população não tratada.

Dois estudos multicêntricos muito bem elaborados afastam a possibilidade de o rhGH aumentar o risco de recorrência da doença tumoral. Um desses estudos avaliou a evolução da neoplasia em 1.071 pacientes com tumores de sistema nervoso central (excluindo craniofaringioma), dos quais 180 tratados com rhGH (77). Outro estudo avaliou 13.539 pacientes que sobreviveram a um câncer infantil, dos quais 361 foram tratados com rhGH e incluiu, além dos pacientes com diagnóstico de tumores de sistema nervoso central, outras patologias como linfomas, leucemias e sarcomas (78). Foi avaliado não apenas o risco de recorrência da doença, mas também um outro aspecto muito importante no tratamento hormonal desses pacientes, $\mathrm{O}$ aparecimento de um segundo tumor. Embora a incidência de leucemia não tenha sido significativa nos pacientes tratados, parece que o risco de aparecimento de um tumor sólido está discretamente aumentado, embora restrito aos sobreviventes de leucemia e linfoma. A importância clínica desse achado ainda é incerta.

Existem outras questões que ainda permanecem sem resposta e não existem estudos com número suficiente de pacientes para se ter absoluta certeza da não interferência do rhGH na recorrência da doença de base. Nessas condições, não se pode considerar que outros tumores, com natureza diversa daquela dos tumores do sistema nervoso, sejam considerados iguais, na vigência de rhGH em relação a esse comportamento, e extrapolar os dados não seria conveniente.

A maioria das crianças tratadas por câncer que apresentam deficiência de GH tem patologia do sistema nervoso central ou doença hematológica tratada com radioterapia. Nesses casos, antes de se iniciar a terapia com rhGH deve-se considerar o comportamento da doença de base, as condições do tumor (ressecado completamente ou não), a probabilidade de recidiva e as condições gerais do paciente. Especial- 
mente em tumores como meduloblastoma e craniofaringioma, que têm alto risco de recidiva, é aconselhável aguardar um período mínimo de 1 ano antes de iniciar a terapia de reposição hormonal. Nas leucemias aparentemente não há a necessidade desse intervalo de tempo (78).

\section{Comentários finais}

Estudos in vitro e em animais sugerem que os membros do sistema IGF promovem a progressão do ciclo celular e inibem a apoptose tanto por ação direta com outros fatores de crescimento como por ação indireta, interagindo com outros sistemas moleculares intracelulares envolvidos na promoção e/ou progressão do câncer. Além disso, inúmeros estudos epidemiológicos têm sugerido que o aumento das concentrações circulantes de IGFs acompanhados ou não às concentrações alteradas das IGFBPs podem estar associados ao risco aumentado de desenvolvimento de determinadas neoplasias. Relatos de que a leucemia é mais freqüente nos pacientes tratados com rhGH não foram confirmados. Existem evidências claras de que o tratamento com rhGH deve ser visto com cautela nos pacientes de risco, tais como os que apresentam fragilidade cromossômica, cromossomopatia, síndromes genéticas, como Down, Bloom e anemia de Fanconi. O tratamento com rhGH não parece aumentar a recorrência do tumor de base desde que este tenha sido adequadamente tratado. Uma pequena porcentagem $(3 \%)$ dos pacientes com câncer tem probabilidade de desenvolver uma segunda neoplasia, e os pacientes com leucemia e linfoma têm um risco discretamente aumentado de desenvolver tumores sólidos.

\section{REFERÊNCIAS}

1. Pollak MN, Schernhammer ES, Hankinson SE. Insulin-like growth factors and neoplasia. Nature Rev Cancer 2004;4:505-18.

2. Foulstone E, Prince $S$, Zaccheo $O$, Burns JL, Harper J, Jacobs C, et al. Insulin-like growth factor ligands, receptors, and binding proteins in cancer. J Pathol 2005; 205 : 145-53.

3. Ibrahim YH, Yee D. Insulin-like growth factor-I and cancer risk. Growth Horm IGF Res 2004; 14:261-9.

4. LeRoith D, Roberts CT Jr. The insulin-like growth factor system and cancer. Cancer Lett 2003;195:127-37.

5. Wilson TA, Rose SR, Cohen $P$, Rogol AD, Backeljauw $P$, Brown $R$, et al. Update of guidelines for the use of growth hormone in children: the Lawson Wilkins Pediatric Endocrinology Society Drug and Therapeutics Committee. J Pediatr 2003;143:415-21.
6. Ogilvy-Stuart AL, Gleeson H. Cancer risk following growth hormone use in childhood. Implications for current practice. Drug Saf 2004;27:369-82.

7. Monzavi R, Cohen P. IGFs and IGFBPs: role in health and disease. Best Pract Res Clin Endocrinol Metab 2002; 16:433-47.

8. Moon HD, Simpson ME, Li CH, Evans HM. Neoplasms in rats treated with pituitary growth hormone. I: pulmonary and lymphatic tissues. Cancer Res 1950;10:297-8.

9. Rogers PC, Komp D, Rogol A, Sabio H. Possible effects of growth hormone on development of acute lymphoblastic leukaemia. Lancet 1977;2:434-5.

10. Jones JI, Clemmons DR. Insulin-like growth factors and their binding proteins: biological actions. Endocr Rev 1995; 16:3-34.

11. Tatar M, Bartke A, Antebi A. The endocrine regulation of aging by insulin-like signals. Science 2003;299:1346-51.

12. Firth SM, Baxter RC. Cellular actions of the insulin-like growth factor binding proteins. Endocr Rev 2002;23:824-54.

13. Clemmons DR. Role of insulin-like growth factor binding proteins in controlling IGF actions. Mol Cell Endocrinol 1998; 140:19-24.

14. Baker J, Liu JP, Robertson EJ, Efstratiadis A. Role of insulin-like growth factors in embryonic and postnatal growth. Cell 1993;75:73-82.

15. Sheppard MC. Growth hormone - from molecule to mortality. Clin Med 2004;4:437-40.

16. Nakae J, Kido Y, Accili D. Distinct and overlapping functions of insulin and IGF-receptors. Endocr Rev 2001;22:818-35.

17. De Meyts $P$, Whittaker J. Structural biology of insulin and IGF-1 receptors: implications for drug design. Nat Rev Drug Discov 2002;1:769-83.

18. Hassan $A B$. Keys to the hidden treasures of the mannose 6-phosphate/insulin-like growth factor 2 receptor. Am J Pathol 2003; 162:3-6.

19. Siddle K, Urso B, Niesler CA, Cope DL, Molina L, Surinya $\mathrm{KH}$, et al. Specificity in ligand binding and intracellular signaling by insulin and insulin-like growth factor receptors. Biochem Soc Trans 2001;29:513-25.

20. Favelyukis S, Hill JH, Hubbard SR, Miller WT. Structure and autoregulation of the insulin-like growth factor 1 receptor kinase. Nature Struct Biol $2001 ; 8: 1058-63$.

21. Sorensen H, Whittaker L, Hinrichsen J, Groth A, Whittaker $J$. Mapping of the insulin-like growth factor II binding site of type I insulin-like growth factor receptor by alanine scanning mutagenesis. FEBS Lett 2004;565:19-22.

22. Baserga R, Peruzzi F, Reiss K. The IGF-1 receptor in cancer biology. Int J Cancer 2003; 107:873-7.

23. Freeman M, Gurdon JB. Regulatory principles of developmental signaling. Ann Rev Cell Dev Biol 2002; 18:515-39.

24. Birkenkamp KU, Coffer PJ. Regulation of cell survival and proliferation by the FOXO (Forkhead box, class 0 ) subfamily of Forkhead transcription factors. Biochem Soc Trans 2003;31:292-7. 
25. Accili D, Arden KC. FOXOs at the crossroads of cellular metabolism, differentiation, and transformation. Cell 2004;117:421-6.

26. Lawlor MA, Alessi DR. PKB/Akt: a key mediator of cell proliferation, survival, and insulin responses? J Cell Sci 2001;114:2903-10.

27. Dupont J, Pierre A, Froment P, Moreau C. The insulin-like growth factor axis in cell cycle progression. Horm Metab Res 2003;35:740-50.

28. Jenkins PJ, Bustin SA. Evidence for a link between IGF-I and cancer. Eur J Endocrinol 2004;151(suppl. 1):S1722.

29. Zhang L, Zhou W, Velculescu VE, Kem SE, Hruban HA, Hamilton SR, et al. Gene expression profiles in normal and cancer cells. Science 1997;276:1268-72.

30. Cui H, Cruz-Correa M, Giardiello FM, Hutcheon DF, Kafonek DR, Bradenburg S, et al. Loss of IGF2 imprinting: a potential marker of colorectal cancer risk. Science 2003;299: 1753-5.

31. Nickerson T, Chang F, Lorimer D, Smeekens SP, Sawyers $\mathrm{CL}$, Pollak M. In vivo progression of LAPC-9 and LNCaP prostate cancer models to androgen independence is associated with increased expression of insulin-like growth factor I (IGF-I) and IGF-I receptor (IGF-IR). Cancer Res $2001 ; 61: 6276-80$.

32. Hellawell GO, Turner GD, Davies DR, Poulsom R, Brewster SF, Macaulay VM. Expression of the type 1 insulin-like growth factor receptor is up-regulated in primary prostate cancer and commonly persists in metastatic disease. Cancer Res 2002;62:2942-50.

33. Pollak M. Insulin-like growth factors (IGFs) and prostate cancer. Epidemiol Rev 2001;23:59-66.

34. Pollak M. Insulin-like growth factor physiology and cancer risk. Eur J Cancer 2000;36:1224-8.

35. Miyake H, Pollak M, Gleave ME. Castration-induced upregulation of insulin-like growth factor binding protein-5 potentiates insulin-like growth factor-I activity and accelerates progression to androgen independence in prostate cancer models. Cancer Res 2000;60:3058-64.

36. Elmlinger MW, Deininger MH, Schuett BS, Meyermman $\mathrm{R}$, Duffner $\mathrm{F}$, Grote $\mathrm{EH}$, et al. In vivo expression of insulinlike growth factor-binding protein in human gliomas increases with tumor grade. Endocrinology $2001 ; 142$ : 1652-8.

37. Baron-Hay S, Boyle F, Ferrier A, Scott C. Elevated serum insulin-like growth factor binding protein-2 as a prognostic marker in patients with ovarian cancer. Clin Cancer Res 2004;10:1796-806.

38. Chan JM, Stampfer MJ, Giovannucci E, Gann PH, Ma J, Wilkinson $\mathrm{P}$, et al. Plasma insulin-like growth factor-l and prostate cancer risk: a prospective study. Science 1998;279:563-6.

39. Hankinson SE, Willett WC, Colditz GA, Hunter DJ, Michaud DS, Deroo B, et al. Circulating concentrations of insulin-like growth factor-l and risk of breast cancer. Lancet 1998;351:1393-6.

40. Gann PH, Hennkens CH, Stampfer MJ. A prospective evaluation of plasma prostate specific antigen for detection of prostate cancer. J Am Med Assoc;273:289-94.

41. Gann PH, Hennkens CH, Ma J, Longcope C, Stampfer

Arq Bras Endocrinol Metab vol $49 n^{\circ} 5$ Outubro 2005
MJ. Prospective study of sex hormone levels and risk of prostate cancer. J Natl Cancer Inst 1996;88:1 1 18-26.

42. Dorgan JF, Longcope C, Stephenson HEJr, Falk RT, Miller $R$, Franz $C$, et al. Serum sex hormone levels are related to breast cancer risk in postmmenopausal women. Environ Health Perspect 1997; 105(suppl. 3):583-5.

43. Harris JR, Lippman ME, Morrow M, Hellman S. Diseases of the breast. Philadelphia:Lippincott-Raven, 1996.

44. Holly JMP, Gunnell DJ, Davey Smith G. Growth hormone, IGF-1 and cancer. Less intervention to avoid cancer? More intervention to prevent cancer? J Endocrinol 1999; 162:321-30.

45. Renehan AG, Zwahlen M, Minder C, O'Dwyer ST, Shalet SM, Egger M. Insulin-like growth factor (IGF)-I, IGF binding protein-3, and cancer risk: systematic review and meta-regression analysis. Lancet 2004;363:1346-53.

46. Harman SM, Metter EJ, Blackman MR, Landis PK, Carter HB. Serum levels of insulin-like growth factor I (IGF-I), IGFII, IGF-binding protein-3, and prostate-specific antigen as predictors of clinical prostate cancer. J Clin Endocrinol Metab 2000;85:4258-65.

47. Stattin P, Bylund A, Rinaldi S, Biessy C, Dechaud H, Stenman UH, et al. Plasma insulin-like growth factor-l, insulinlike growth factor binding proteins, and prostate cancer risk: a prospective study. J Natl Cancer Inst 2000;92:1910-7.

48. Wolk A, Mantzoros CS, Andersson SO, Bergstrom R, Signorello LB, Lagiou P, et al. Insulin-like growth factor I and prostate cancer risk: a population-based, case-control study. J Natl Cancer Inst 1998;90:911-5.

49. Finne $P$, Auvinen A, Koistinen H, Zhang WM, Maattanen L, Rannikko S, et al. Insulin-like growth factor I is not a useful marker of prostate cancer in men with elevated levels of prostate-specific-antigen. J Clin Endocrinol Metab 2000;85:2744-7.

50. Chokkalingam AP, Pollak M, Fillmore CM, Gao YT, Stanczyk FZ, Deng J, et al. Insulin-like growth factors and prostate cancer: a population based case-control study in China. Cancer Epidemiol Biomarkers Prev 2001;10:421-7.

51. Ma J, Pollak M, Giovannucci E, Chan JM, Tao Y, Hennekens $\mathrm{CH}$, et al. Prospective study of colorectal cancer risk in men and plasma levels of insulin-like growth factor (IGF)-I and IGF-binding protein-3. J Natl Cancer Inst 1999;91:620-5.

52. Giovannucci E, Pollak M, Platz EA, Willett WC, Stampfer MJ, Majeed N, et al. A prospective study of plasma insulin-like growth factor-1 and binding protein-3 and risk of colorectal neoplasia in women. Cancer Epidemiol Biomarkers Prev 2000;9:345-9.

53. Kaaks R, Toniolo P, Akhmedkhanov A, Lukanova A, Biessy $C$, Dechaud $H$, et al. Serum-C peptide, insulin-like growth factor (IGF)-I, IGF-binding proteins, and colorectal cancer risk in women. J Natl Cancer Inst 2000; 92:1592-600.

54. Probst-Hensch NM, Yuan JM, Stancyk FZ, Gao YT, Ross RK, Yu MC. IGF-1, IGF-2 and IGFBP-3 in prediagnostic serum: association with colorectal cancer in a cohort of Chinese men in Shanghai. Br J Cancer 2001;85:1695-9.

55. Palmqvist R, Hallmans $G$, Rinaldi S, Biessy $C$, Stenling R, Riboli $E$, et al. Plasma insulin-like growth factor 1 , insulin- 
like growth factor binding protein 3 , and risk of colorectal cancer: a prospective study in northern Sweden. Gut 2002;50:642-6

56. Toniolo P, Bruning PF, Akhmedkhanov A, Bonfrer JM, Koenig KL, Lukanova A, et al. Serum insulin-like growth factor-I and breast cancer. Int J Cancer 2000;88:82832.

57. Muti P, Quatrin T, Grant BJ, Krogh V, Micheli A, Schunemann $\mathrm{HJ}$, et al. Fasting glucose is a risk factor for breast cancer: a prospective study. Cancer Epidemiol Biomarkers Prev 2002;11:705-12.

58. Krajcik RA, Borofsky ND, Massardo S, Orentreich N. Insulin-like growth factor I (IGF-I), IGF-binding proteins, and breast cancer. Cancer Epidemiol Biomarkers Prev 2002;11:1566-73.

59. Del Giudice ME, Fantus IG, Ezzat S, McKeown-Eyssen G, Page D, Goodwin PJ. Insulin and related factors in premenopausal breast cancer risk. Breast Cancer Res Treat 1998;47:111-20.

60. Yu H, Jin F, Shu XO, Li BD, Dai Q, Cheng JR, et al. Insulinlike growth factors and breast cancer risk in Chinese women. Cancer Epidemiol Biomarkers Prev 2002; 11:705-12.

61. Lukanova A, Toniolo P, Akhmedkhanov A, Biessy C, Haley NJ, Shore RE, et al. A prospective study of insulinlike growth factor-I, IGF-binding proteins-1, -2 and -3 and lung cancer risk in women. Int J Cancer 2001;92:88892.

62. London SJ, Yuan JM, Travlos GS, Gao YT, Wilson RE, Ross RK, et al. Insulin-like growth factor I, IGF-binding protein 3 , and lung cancer risk in a prospective study of men in China. J Natl Cancer Inst 2002;94:749-54.

63. Spitz MR, Barnett MJ, Goodman GE, Thornquist MD, Wu $X$, Pollak $M$. Serum insulin-like growth factor (IGF) and IGF-binding protein levels and risk of lung cancer: a case-control study nested in the beta-Carotene and Retinol Efficacy Trial Cohort. Cancer Epidemiol Biomarkers Prev 2002;11:1413-8.

64. Yu H, Spitz MR, Mistry J, Gu J, Hong WK, Wu X. Plasma levels of insulin-like growth factor-l and lung cancer risk: a case-control analysis. J Natl Cancer Inst 1999;91:151-6.

65. Woodson K, Tangrea JA, Pollak M, Copeland TD, Taylor PR, Virtamo J, et al. Serum insulin-like growth factor I: tumor marker or etiologic factor? A prospective study of prostate cancer among Finish men. Cancer Res 2003:63:3991-4.

66. Juul A, Scheike T, Davidsen M, Gyllenborg J, Jörgensen T. Low serum insulin-like growth factor I is associated with increased risk of ischemic heart disease. A population-based case-control study. Circulation 2002; 106:939-44.

67. Sandhu MS, Heald AH, Gibson JM, Cruickshank JK, Dunger DB, Wareham NJ. Circulating concentrations of insulin-like growth factor-l and development of glucose intolerance: a prospective observational study. Lancet 2002;359:1740-5.
68. Blethen SL. Leukemia in children treated with growth hormone. Trends Endocrinol Metab 1998:9:367-70.

69. Robertson CM, Hawkins MM, Kingston JE. Late deaths and survival after childhood cancer: implications for cure. Br Med J 1994;309:162-6.

70. Allen DB, Rundle AC, Graves DA, Blethen SL. Risk of leukemia in children with growth hormone: review and reanalysis. J Pediatr 1997;131:S32-6.

71. Blethen SL, Allen DG, Graves D, August G, Moshang T, Rosenfeld R. Safety of recombinant deoxyribonucleic acid-derived growth hormone: the national cooperative growth study experience. J Clin Endocrinol Metab 1995;81:1704-10.

72. Growth Hormone Research Society. Critical evaluation of the safety of recombinant human growth hormone administration: statement. J Clin Endocrinol Metab $2001 ; 86$ : 1868-70.

73. Nishi $Y$, Tanaka T, Takano K, Fujieda K, Igarashi $Y$, Watanabe $S$, et al. Recent status in the occurrence of leukemia in growth hormone-treated patients in Japan. J Clin Endocrinol Metab 1999;84:1961-5.

74. Tufflli GA, Johanson A, Rundle AC, Allen DB. Lack of increased risk for extracranial, nonleukemic neoplasms in recipients of recombinant deoxyribonucleic acid growth hormone. J Clin Endocrinol Metab 1995,80: 1416-22.

75. Swerdlow JA, Higgins DC, Adlard P, Preece MA. Risk of cancer in patients treated with human pituitary growth hormone in the UK, 1959-85: a cohort study. Lancet 2002;360:273-7.

76. Lawson-Wilkins Pediatric Endocrine Society (LWPES) Writing Committee. Special editorial: growth hormone treatment and neoplasia - coincidence or consequence? J Clin Endocrinol Metab 2002;87:5351-2.

77. Swerdlow AJ, Reddingius RE, Higgins CD, Spoudeas HA, Phipps K, Qiao Z, et al. Growth hormone treatment of children with brain tumors and risk of tumor recurrence. J Clin Endocrinol Metab 1995;81:1704-10.

78. Sklar AC, Mertens AC, Mitby P, Ochiogrosso G, Quin J, Robinson $L$, et al. Risk of disease recurrence and second neoplasms in survivors of childhood cancer treated with growth hormone: a report from the childhood cancer survivor study. J Clin Endocrinol Metab 2002;87:5351-2.

\section{Endereço para correspondência:}

Gil Guerra-Júnior

Departamento de Pediatria

FCM - UNICAMP

CP 6111

13083-970 Campinas, SP

Fax: (19) 3788-7322

E-mail: gilguer@fc.unicamp.br 